\title{
The Neighborhood Effects of a Place-Based Policy-Causal Evidence from Atlanta's Economic Development Priority Areas
}

\author{
Yuxi Luo ${ }^{1}$, Zhaohua Zhang ${ }^{2, *}$, Jun Zheng ${ }^{2, *}$ and Diane Hite ${ }^{3}$ \\ 1 School of Economics and Management, Guangxi Normal University, Guilin 541004, China; \\ yluogxnu@mailbox.gxnu.edu.cn \\ 2 College of Economics and Management, Shandong Agricultural University, Tai'an 271018, China \\ 3 Department of Agricultural Economics and Rural Sociology, Auburn University, Auburn, AL 36849, USA; \\ hitedia@auburn.edu \\ * $\quad$ Correspondence: zzhang@sdau.edu.cn (Z.Z.); jzheng_sdau@163.com (J.Z.); Tel.: +86-0538-8246378 (Z.Z.)
}

Citation: Luo, Y.; Zhang, Z.; Zheng, J.; Hite, D. The Neighborhood Effects of a Place-Based Policy-Causal Evidence from Atlanta's Economic Development Priority Areas. Sustainability 2021, 13, 6808. https:// doi.org/10.3390/su13126808

Academic Editors: Bharat Dahiya and Jonathan Parkinson

Received: 20 May 2021

Accepted: 11 June 2021

Published: 16 June 2021

Publisher's Note: MDPI stays neutral with regard to jurisdictional claims in published maps and institutional affiliations.

Copyright: ( $\odot 2021$ by the authors Licensee MDPI, Basel, Switzerland. This article is an open access article distributed under the terms and conditions of the Creative Commons Attribution (CC BY) license (https:// creativecommons.org/licenses/by/ $4.0 /)$.

\begin{abstract}
Place-based policies refer to government efforts to enhance the economic performance of an area within its jurisdiction. Applying various difference in differences strategies, this study evaluates the neighborhood effects of a place-based policy—the Economic Development Priority Areas (EDPA) of Atlanta, Georgia, USA. Since the census block groups are locally defined and the boundaries may change over time, we defined the neighborhoods by creating a set of 0.25 -mile- diameter circles evenly distributed across Atlanta, and used the created buffers as the comparison unit. The empirical estimates showed that EDPA designation significantly reduced poverty rate and increased housing price of EDPA neighborhoods but had no beneficial effects on population size and employment rate. The heterogeneous analysis with respect to different initial economic status of the neighborhoods showed a relative larger and significant effect of EDPA designation on low-income neighborhoods. The increasing labor demand induced by EDPA designation in low-income neighborhoods attracted more population to migrate in and put upward pressure on housing prices. The estimation results are robust when replacing the 0.25 -mile-diameter circle neighborhoods with 0.5 -mile-diameter circle neighborhoods. Although we found some positive effects of the EDPA program in Atlanta, it would be misguided to assume similar effects occur in other areas implementing place-based policies.
\end{abstract}

Keywords: enterprise zone; difference in difference model; propensity score weighting; placebased policy

\section{Introduction}

In the past two decades, place-based policies have been widely accepted by local, state, and federal policy makers to encourage private development and redevelopment in areas which otherwise would not be developed due to the existence of certain characteristics of the area (such as low capital investment, falling demand for labor, etc.). These place-based policies often offered spatially targeted tax abatements, subsidies, public investments, and regulations [1]. Kline and Moretti (2014) [2] noted that spending on place-based policies has outpaced more traditional person-based assistance such as unemployment insurance. Enterprise Zone programs quickly became one of the most popular place-based polices. The City of Atlanta's Urban Enterprise Zone Program was authorized for creation by the Georgia General Assembly in 1983. The Economic Development Priority Areas (EDPA) of Atlanta were identified in 2005, which is a part of the New Century Economic Development Plan. Property within these EDPAs, which are Campbellton Road, Donald Lee Hollowell Parkway, Jonesboro Road, Memorial Drive Corridor, Simpson Road, and Stadium Neighborhoods, are automatically eligible for Urban Enterprise Zone designation. These six areas were once thriving commercial corridors and they have suffered from years of neglected and disinvestment (Guide to the City of Atlanta's Urban Enterprise Zone Program, online at http: / / www.atlantaga.gov /index.aspx?page=326, accessed on 
17 August 2015). The rationale guiding policy makers for designing these areas is that reductions in taxation are meant to offset the numerous disadvantages associated with deprived areas, such as a shortage of skilled labor, a lack of public services, a dearth of inputs, or poor market potential [3]. However, by reviewing a large body of economic literature on place-based policies, Neumark and Simpson (2014) [4] showed that the theoretical foundations of such policies were not well established, and the empirical evidence on their efficiency was mixed. In this study, we present new empirical evidence on the effectiveness of place-based policies using Atlanta's Urban Enterprise Zone Program as an example, which may provide design guidance for a more effective program.

Many countries have widely employed place-based policies to narrow the considerable development gaps across regions [5]. In the United States, approximately USD 95 billion per year have been spent on place-based policies in the first decade of the 21st century [6]. Such policies are often justified on the grounds that temporarily making a region attractive may convince firms to move, creating a new center of agglomeration that remains productive after the policies end [7]. Research examining Enterprise Zones has predominately focused on affected businesses. Topics have included job creation and local economic activities. Studies on French Urban Zones program have found it had at best modest and heterogeneous effects on employment [3,8]. O'Keefe (2004) [9] and Neumark and Kolko (2010) [10] both study California's enterprise zone program but draw opposite conclusions. Bondonio and Engberg (2000) [11], who study the State Enterprise Zones, find no effect, whereas Busso et al. (2008) [12] find that neighborhoods receiving federal empowerment zones experienced substantial improvements in labor market conditions. With respect to local economic activities, Lambert and Coomes (2001) [13] took a quasi-experimental approach in evaluating Louisville's enterprise zone, and found little evidence of net new economic activity in the initially targeted, truly distressed zones; Zhang (2015) [14] evaluates the impacts of the Louisville enterprise zone program on the growth of different industries, and results suggest that the Louisville enterprise zone program has significantly increased the growth of manufacturing and service activities. All these studies differ from each other in outcome measurements, spatial unit, and modeling methodology.

This paper contributes to the literature concerned with whether place-based policies have stimulated economic growth of the neighborhoods [7,15-17]. While a growing body of literature evaluated the effectiveness of enterprise zone programs, mixed conclusions about program effectiveness were provided $[18,19]$. A review of the existing literature suggests that more appropriate spatial units and better data may affect conclusions on the policy evaluation. This study offers a post-program evaluation of the Atlanta's EDPA program, with the following improvements. First, comparing with previous studies, this paper creates neighborhoods by drawing a set of 0.25 -mile-radius circles across the whole city of Atlanta, Georgia (GA). Then, key outcome variables are attached to the new neighborhoods based on the percentage of the block group's geographic area lying within each circle. The benefit of this approach is that we need not to worry about the change of neighborhood boundaries from 2000 to 2010, and the same sized neighborhoods reduced bias when averaging outcome variables over different areas. Furthermore, existing literature evaluating enterprise zone programs has predominately focused on topics such as labor market outcomes $[9,10,20,21]$, business location decisions $[3,16]$, and changes to commercial and industrial property values [22]. Besides the effect on local labor market, this study also assesses the impact of the EDPAs on residential sorting behavior and local housing market using detailed data on sale prices of Atlanta residential properties. If EDPAs are successful, population size of the neighborhoods would increase, and the local real estate market should reflect the increased demand of houses for living and working, which would raise the market value of housing. An increase in housing values in EDPAs compared with similar non-EDPAs would be evidence that enterprise zones are having a positive impact. Additionally, the raised values should help increase local wealth and thus 
the tax base, reduce abandonment, and help improve the attractiveness of purchasing a house [23].

In line with the usual practice in the empirical literature on program effectiveness, the difference in difference strategy is applied, which allows us to compare outcome variables between EDPA neighborhoods and non-EDPA neighborhoods along with the timing of the NEDPA implementation. Estimation results from the naïve difference in difference analysis (DID), propensity score DID, and the Blinder-Oaxaca DID are reported in this study. The basic idea of the propensity score DID is to reweight the data in a manner that balances the distribution of covariates across treated and untreated tracts, and with balanced distribution of covariates, a simple comparison of weighted means will identify the treatment effect of the EDPA program. However, the procedure of Blinder-Oaxaca DID is first to impute the counterfactual outcomes for the treated neighborhoods, and then apply the average difference between the imputed values and actual values among treated tracts as an estimator of treatment effect of DEPA program. Combining all three approaches, our empirical results showed that EDPA designation significantly reduced poverty rate and increased housing price of EDPA neighborhoods. However, our results failed to find beneficial effects of EDPA program on population size, employment rate, and household income. To further test whether impacts of DEPA designation vary with the initial economic status of the neighborhoods, we divided the neighborhoods into lowincome neighborhoods, medium-income neighborhoods, and high-income neighborhoods based on their percentile (25\%, 75\%, and above) in the 2000 median household income distribution. Our results showed a relative larger and significant effect of EDPA designation on low-income neighborhoods. With increasing labor demand induced by EDPA designation in low-income neighborhoods, we found that more population migrated in these neighborhoods, putting upward pressure on housing prices. To test whether our conclusions are sensitive to neighborhood size, following Banzhaf and Walsh (2008) [24], we alternatively define the neighborhoods with a larger size. The estimation results are robust when replacing the 0.25 -mile-diameter circle neighborhoods with 0.5 -mile-diameter circle neighborhoods. The rest of this study is structured as follows: Section 2 provides details of the methodology used to assess the impacts of the EDPAs. Housing price data and other data sources are described in Section 3. Section 4 reports the results and discussion of this analysis, and Section 5 gives conclusions.

\section{Econometric Model}

The econometric model used in this paper closely follows the model developed by Busso et al. (2008) [12]. Let outcomes of neighborhood $i$ in time period $t$ be represented by $Y_{i t}$. These outcomes are generated by a model of the following form:

$$
Y_{i t}=\mu_{t}\left(T_{i t}, Y_{i t-1}, X_{i t-1}, \varepsilon_{i t}\right)+\theta_{i}
$$

where $\mu_{t}(\cdot)$ is a function indexed by time, $T_{i t}$ is a treatment dummy, $Y_{i t-1}$ is the neighborhood outcome lagged, $X_{i t-1}$ is a vector of predetermined neighborhood characteristics, $\theta_{i}$ is a neighborhood fixed effect, and $\varepsilon_{i t}$ is the error term, which is assumed to be independent of all other right-hand-side variables. In convenience, we reexpress the dependence of the function $u_{t}\left(T_{i t}.\right)$ on EDPA designation by writing the following equation:

$$
u_{t}\left(T_{i t}, .\right)=T_{i t} \mu_{t}^{1}(\cdot)+\left(1-T_{i t}\right) \mu_{t}^{0}(\cdot)
$$

The effect of EDPA designation on outcomes in a given neighborhood could be now defined as:

$$
\beta_{i}=\mu_{t}^{1}(\cdot)-\mu_{t}^{0}(\cdot)
$$

Note that this effect of a potentially nonlinear function of the predetermined covariates $Y_{i t-1}$ and $X_{i t-1}$. This indicates that neighborhoods with different preexisting characteristics are likely to exhibit different responses to EDPA designation. In order to eliminate the 
neighborhood fixed effect $\theta_{i}$, we rewrite Equation (1) in first differences using the outcomes notion propose by Splawa-Neyman (1990) [25] and Rubin (1974) [26]:

$$
\begin{gathered}
\Delta Y_{i t}^{1}=\beta_{i}+h_{t}\left(\Omega_{i t}, U_{i t}\right) \\
\Delta Y_{i t}^{0}=h_{t}\left(\Omega_{i t}, U_{i t}\right)
\end{gathered}
$$

where $h_{t}(\cdot)=\mu_{t}^{0}(\cdot)-\mu_{t-1}^{0}(\cdot), \Omega_{i t}=\left(Y_{i t-1}, X_{i t-1}, Y_{i t-2}, X_{i t-2}\right)$, and $U_{i t}=\left(\varepsilon_{i t}, \varepsilon_{i t-1}\right)$. Superscripts index potential outcomes under different treatment status. Since we have only one post-treatment period, outcomes associated with two time periods of EDPA designation or one period are not considered in this study and static treatment schemes are applied. $\Delta Y_{i t}^{1}$ represents the change in $Y_{i t}$ a neighborhood would have experienced with an EDPA, while $\Delta Y_{i t}^{0}$ represents the change that would occurred without an EDPA. Since we could only observe one of these potential outcomes for each neighborhood, we may write:

$$
\Delta Y_{i t}=\Delta Y_{i t}^{1} T_{i t}+\Delta Y_{i t}^{0}\left(1-T_{i t}\right)
$$

Since the neighborhood specific impact of DEPAs designation $\beta_{i}$ is itself a function of neighborhood characteristics. The average effect of treatment on the treated neighborhoods, which may be defined as:

$$
\tau=E\left[\Delta Y_{i t}^{1}-\Delta Y_{i t}^{0} \mid T_{i t}=1\right]=E\left[\beta_{i} \mid T_{i t}=1\right]
$$

The average effect of treatment on the treated measures the average impact of the EDPA on those neighborhoods received the EDPAs designation. To estimate $\tau$, we need to identify two factors: $E\left[\Delta Y_{i t}^{1} \mid T_{i t}=1\right]$ and $E\left[\Delta Y_{i t}^{0} \mid T_{i t}=1\right]$. The first factor can be identified directly by the sample mean of the treated observations on $\Delta Y_{i t}$. However, the second factor is the counterfactual mean of the treated observations had they not been treated, which we have no observable samples. To solve this problem, two proposed approaches are applied. The first approach, which is a variant of Blinder (1973) [27] and Oaxaca (1973)'s [28] approach to decomposing wage distributions, is to estimate the function of $E\left[\Delta Y_{i t}^{0} \mid \Omega_{i t}, T_{i t}=0\right]$ using a parametric model and then to use the estimated model to compute an approximate of $E\left[\Delta Y_{i t}^{0} \mid T_{i t}=1\right]=\int E\left[\Delta Y_{i t}^{0} \mid \Omega_{i t}, T_{i t}=0\right] d F\left(\Omega_{i t} \mid T_{i t}=1\right)$. We do this by fitting a flexible regression model to the untreated neighborhoods and use the estimated coefficients to impute the counterfactual mean outcomes of each treated neighborhoods. Thus, for each neighborhood we have a specific treatment effect $\hat{\beta}_{i}=$ $\Delta Y_{i t}^{1}-\Delta \hat{Y}_{i t}^{0}\left(\Omega_{i t}\right)$, where $\Delta \hat{Y}_{i t}^{0}\left(\Omega_{i t}\right)=\hat{E}\left(\Delta Y_{i t}^{0} \mid \Omega_{i t}\right)$ is the prediction from a parametric linear regression function. Then, the average treatment effect can be defined as:

$$
\tau_{B O}=\frac{1}{N_{1}} \sum_{i \in\{D=1\}} \hat{\beta}_{i}
$$

The second approach is to approximate the counterfactual mean $E\left[\Delta Y_{i t}^{0} \mid D_{i t}=1\right]$ through propensity score reweighting, which is proposed by Horvitz and Thompson (1952) [29]. Propensity score approach could reduce the bias of estimating the treatment effect obtained from simply comparing outcomes among treated group and untreated group. The basic idea of the propensity score approach is to balance the distribution of covariates across treated neighborhoods and untreated neighborhoods. After the distribution of the covariates is balanced, the average treatment effect can be easily identified. To use propensity score weighting, it is necessary to assume that (1) selection into a treatment is a function of $\Omega_{i t}, T_{i t}=1$ if $T_{i t}^{*}>0$ and 0 otherwise, where $T_{i t}^{*}=\lambda \Omega_{i t}+v_{i t} . \lambda$ is the coefficient and $v_{i t}$ is a random error; $(2) v_{i t} \perp\left(\Omega_{i t}, U_{i t}\right)$ (the conditional independence condition); and (3) $P\left(T_{i t}=1 \mid \Omega_{i t}\right)<1$, which is often referred to as the "common support" condition. 
These detailed conditions are described by Heckman et al. (1998) [30]. With propensity score reweighting we have:

$$
E\left[\Delta Y_{i t}^{0} \mid T_{i t}=1\right]=E\left[\omega\left(\Omega_{i t}\right) \Delta Y_{i t}^{0} \mid T_{i t}=0\right]
$$

where $\omega\left(\Omega_{i t}\right)=\frac{p\left(\Omega_{i t}\right)}{1-p\left(\Omega_{i t}\right)} * \frac{1-\pi}{\pi}, p\left(\Omega_{i t}\right)=P\left(T_{i t}=1 \mid \Omega_{i t}\right)$, and $\pi=P\left(T_{i t}=1\right)$. As a result, the untreated group can be made to mimic that of the treated group by weighting observations by $\omega\left(\Omega_{i t}\right)$. Equation (8) simplifies the estimation considerably since rather than estimating a very high dimensional conditional expectation, we just need to estimate the propensity score $p\left(\Omega_{i t}\right)=P\left(T_{i t}=1 \mid \Omega_{i t}\right)$ [31]. In empirical estimation, $p\left(\Omega_{i t}\right)$ is estimated via a logit regression and $\pi$ is calculated by the fraction of treated neighborhoods in the sample, which can be expressed as $\frac{N^{1}}{N^{0}+N^{1}}$.

After $p\left(\Omega_{i t}\right)$ and $\pi$ is estimated, using the sample data $E\left[\omega\left(\Omega_{i t}\right) \Delta Y_{i t}^{0} \mid T_{i t}=0\right]$ can be estimated as follows:

$$
E\left[\omega\left(\Omega_{i t}\right) \Delta Y_{i t}^{0} \mid T_{i t}=0\right]=\frac{1}{N^{0}} \sum \frac{\hat{p}\left(\Omega_{i t}\right)}{1-\hat{p}\left(\Omega_{i t}\right)} * \frac{1-\hat{\pi}}{\hat{\pi}} * \Delta Y_{i t}^{0}
$$

Then, the average treatment effect can be estimated as:

$$
\tau_{p s}=\frac{1}{N^{1}} \sum_{i \in\{T=1\}} \Delta Y_{i t}^{1}-\frac{1}{N^{0}} \sum_{i \in\{T=0\}} \sum \frac{\hat{p}\left(\Omega_{i t}\right)}{1-\hat{p}\left(\Omega_{i t}\right)} * \frac{1-\hat{\pi}}{\hat{\pi}} * \Delta Y_{i t}^{0}
$$

Throughout the paper we showed estimation results for both the Blinder-Oaxaca and propensity score reweighting approaches.

\section{Data Sources}

\subsection{Definition of Neighborhoods}

The study area in this paper is the Atlanta, GA. In order to get the treatment effects of EDPAs designation on neighborhoods using difference-in-difference analysis, it is required that the boundaries of the study units in both 2000 and 2010 remained fixed. In previous studies (see Numark and Young, 2019; Ham et al., 2011) [17,32], census block groups or census tracts were often used as study unit. However, census block groups or census tracts are locally defined to create relatively homogenous entities, and boundaries of many census block groups or census tracts had changed between decennial censuses. The red lines in Figure 1 represent the boundaries of the 2000 census block group of Atlanta, while the green lines show the boundaries of the 2010 census block group. From Figure 1 we can see that the red lines and green lines are not perfectly overlapped, which means that the boundaries of the census block group changed during 2000 and 2010. Another problem of using census block groups or census tract as the study unit is that the size of the unit is different, and the quality of outcome variables may be lower when averaged over a large area, which may bias the results. Therefore, following Banzhaf and Walsh (2008) [24], this study takes a different approach for neighborhood definitions. We define our neighborhoods as a set of 0.25-mile-diameter circles (alternatively 0.5-mile-diameter circles) evenly distributed across our study area. The process of new neighborhoods construction is as follows. First, an equidistant grid was placed across the Atlanta, GA, using the ArcGIS software. Both the width and height of the grids is 0.25 mile. After the grids have been constructed, a 0.125 mile buffer was placed inside each grid, creating a set of circles of 0.25 -mile diameter that were evenly distributed within the boundaries of our study area (see Figure 2, the square in the upper left corner shows a partial enlarged view of Figure 2). This process creates 2023 “0.25-mile- diameter neighborhoods". Since 0.25-mile-diameter circles are applied in this study, the gaps among the circles are very small (see Figure 2), and we neglected these gaps in the analysis. Though regular grids are frequently used in geographical studies, circles are preferred in this study due to several advantages. Compared with 
grids, circles have shorter perimeter than a square of equal area, which potentially reduces bias due to edge effects. Another important advantage of the circles is the unambiguous definition of nearest neighborhood: each circle has four adjacent circles in symmetrically equivalent positions; in contrast, the rectangular grid has two different kinds of nearest neighbor: orthogonal neighbors sharing an edge and diagonal neighbors sharing only a corner. Defining neighborhoods with distance to the nearest EDPA area less than 0.25 mile as treated groups in this study, variables calculations on rectangular grids require a setting for the relative weighting of diagonal interactions, which is avoided by using circles [33].

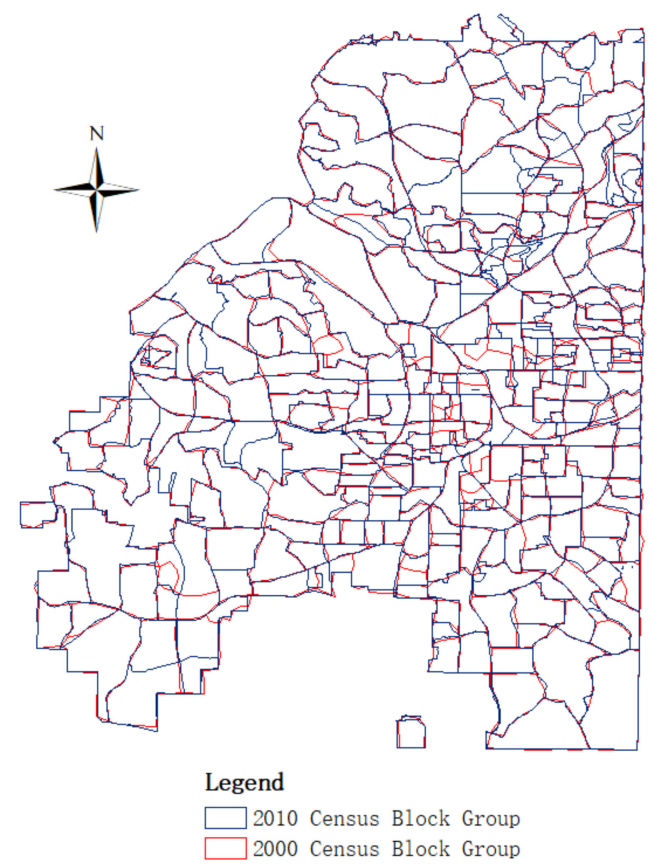

Figure 1. Changes of Census Block Group between 2000 and 2010.

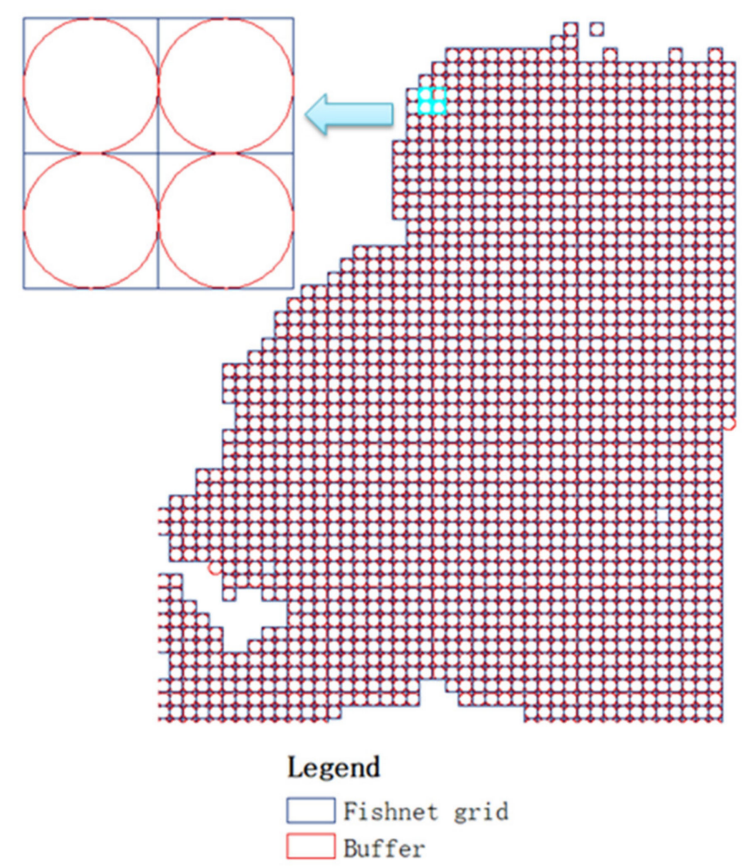

Figure 2. New Neighborhoods Creation across Atlanta.

With the help of the ArcGIS software, demographic data from census block groups and housing price information then can be attached to the new neighborhoods based on the 
percentage of the block group's geographic area lying within each neighborhood. Figure 3 shows the approach used to assign data to the new neighborhoods across the study area. In Figure 3, the blue circles represent the new neighborhoods, and the green lines represent the boundaries of the census block groups. Therefore, data are assigned to the neighborhoods based on the percentage of the geographic area within the dark blue boundaries overlap with each circle. Taking population for example, population from four census block groups are assigned to the selected new neighborhood (light blue circle): $33.16 \%$ of the population in census block group " 131210042001 ", $3.07 \%$ of the population in census block group "131210043001", $2.01 \%$ of the population in census block group " 131210039002 ", and $2.30 \%$ of the population in census block group "131210042002". Similarly, all other demographic data from the 2000 and 2010 census are aggregated to the new circle-neighborhoods. The demographic characteristics for each neighborhood are easily comparable because of the same size (0.19625 square miles).

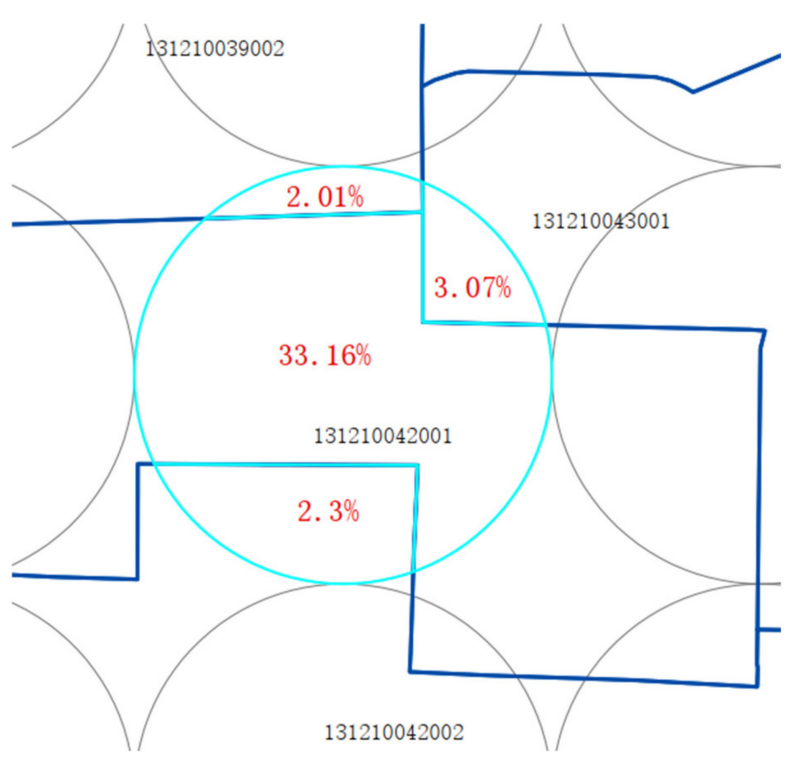

Figure 3. Variable Assignment.

\subsection{Housing and Neighborhood Characteristics}

The American enterprise zone policies have usually attempted to assist community as well as industrial development. This differs from policies in the European countries, where the primary focus was reviving abandoned industrial areas. Although most of the enterprise zone programs in the United States are aimed at attracting and retaining jobs, the ultimate goal is community revitalization [23]. Therefore, the outcome variables of the neighborhood in this study include population size, percentage of black population, employment rate, median household income, poverty level, and housing price. To perform the treatment analysis of EDPAs, we constructed a detailed panel dataset combining information from the Decennial Census and real estate transaction data of Atlanta, GA. The EDPAs in Atlanta are shown in Figure 4. Data about population size, percentage of black population, employment rate, average household income, and poverty level are first collected at the block-group level from the census, and then assigned to our circle neighborhoods based on the percentage of the block group's geographic area lying within each circle. Since the EDPAs were identified in 2005, our study period is from 2000 to 2010 to do the pre-program and post-program comparison. Census block group data about population, employment rate, poverty rate, and median household income are drawn from the 2000 and 2010 Decennial Censuses. The housing data include residential real estate transaction data of Atlanta, GA, which are provided by Fulton County Assessor's Office. We only focus on single-family residential properties transacted in 2000 and 2010. The property data provide transaction records for residential properties located in Atlanta. Each record 
includes the property's address, transaction price and the structure characteristics of the houses. Each transaction is assigned to a neighborhood based on its geographic location.

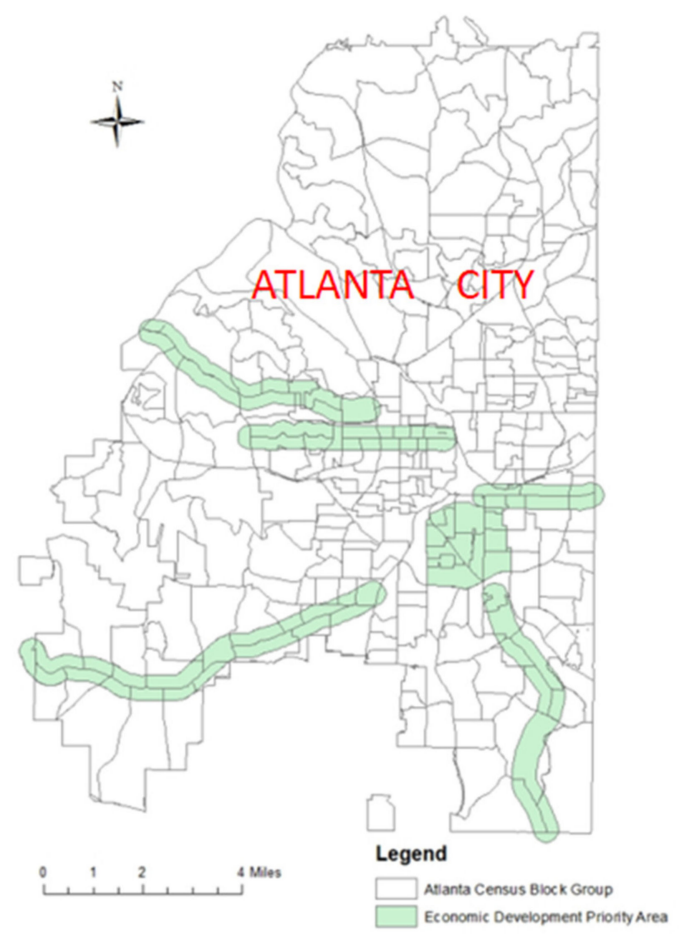

Figure 4. Economic Development Priority Areas.

\section{Summary Statistics and Empirical Results}

\subsection{Summary Statistics of Key Variables}

In this study, we use the created neighborhoods as the unit to explore the changes in outcomes. We keep the neighborhoods with non-missing values for all the outcome variables in our sample. The treatment group in our study contains neighborhoods located within the EDPAs. Table 1 provides descriptive statistics of key variables for neighborhoods of both the EDPA and the non-EDPA neighborhoods. A few facts can be concluded from Table 1. First, from the initial conditions of the neighborhoods in 2000 we can see that the median household income, employment rate, and housing price in 2000 are much lower in EDPA neighborhoods than those in non-EDPA neighborhoods. However, the percentage of black population and poverty level are higher in EDPA neighborhoods. This is consistent with the fact that enterprise zone program is only provided to areas that are less developed. The changes of neighborhood characteristics indicate that share of the black population decreased during the study period, no matter in the EDPA or in the non-EDPA neighborhoods. However, the share of black population decreased more in non-EDPA neighborhoods. Though housing price increased throughout the whole city, there was a larger increase in the EDPA neighborhoods. Employment rate and poverty level changed slightly during the study period in non-EDPA neighborhoods. In the EDPA neighborhoods, poverty rate decreased by $16.5 \%$ and employment rate increased by $4.13 \%$. Median household income increased in both EDPA and non-EDP neighborhoods, but growth in non-EDPA neighborhoods was larger. Note that these descriptive statistics differ from the true causal effect of EDPA designation, because the means capture not only the EDPA effect, but also any trends regarding cohort heterogeneity. 
Table 1. Summary Statistics and Changes of Outcome Variables from 2000 to 2010.

\begin{tabular}{ccccccc}
\hline & \multicolumn{3}{c}{ EDPAs (Mean Values) } & \multicolumn{3}{c}{ Non-EDPAs (Mean Values) } \\
\hline Variables & $\mathbf{2 0 0 0}$ & $\mathbf{2 0 1 0}$ & Change (\%) & $\mathbf{2 0 0 0}$ & $\mathbf{2 0 1 0}$ & Change (\%) \\
\hline Population Size & 176.6 & 161.2 & -8.720 & 170.1 & 186.7 & 9.759 \\
Share Black & 0.906 & 0.837 & -7.616 & 0.583 & 0.533 & -8.576 \\
Employment Rate & 0.464 & 0.484 & 4.132 & 0.598 & 0.593 & -0.836 \\
Median Income (\$1000) & 28.08 & 34.53 & 22.97 & 49.35 & 64.11 & 29.91 \\
Poverty Level & 0.303 & 0.253 & -16.50 & 0.187 & 0.191 & 2.139 \\
Housing Price $(\$ 1000)$ & 81.10 & 92.37 & 12.20 & 172.1 & 190.4 & 9.611 \\
\hline
\end{tabular}

Note: Poverty level is measured by the percentage of population whose poverty status was determined in the last 12 months.

Guerrieri et al. (2012) [34] indicated that impact of enterprise zone programs varied with the initial economic status of the communities. Therefore, to have a better understanding of our data, we first segment the neighborhoods in our dataset by their initial median household income in 2000, and analyze changes of outcome variables in groups with different initial household income. Specifically, we divide the neighborhoods into three groups (low-income neighborhoods, medium-income neighborhoods, and highincome neighborhoods), each representing their percentile (25\%, $75 \%$, and above) in the 2000 median household income distribution. Results are shown in Table 2. First, we come to population size and percentage of black population. For the EDPAs, there was a population decrease in medium-income groups while the population in the low- and high-income groups increased during the study period. This indicates that the EDPAs gain most of the population from the initially poor and rich neighborhoods. For the non-EDPAs, population in low-income neighborhoods changed little, while medium and high income neighborhoods experienced population gains. Most population in the EDPA neighborhoods were black, while richer neighborhoods were occupied by white among the non-EDPA neighborhoods. Percentage of the black population decreased in all three groups in EDPA neighborhoods, and low-income neighborhoods undergo the largest decrease. For non-EDPA neighborhoods, the share of the black population decreased in low- and medium-income groups, but high income neighborhoods experienced an influx of black residents. This means that the percentage of the black population decreased during 2000 to 2010 in all of Atlanta. Comparing the change of the share of the black population in EDPA neighborhoods with that in non-EDPA neighborhoods, we found that low- and medium-income neighborhoods in non-EDPAs experienced larger decrease, indicating that the designation of DEPAs attracted more black population to the low- and medium-income neighborhoods under the situation of decreasing black population in all of Atlanta. The change of employment rate shows that more job opportunities were created for the poorer neighborhood in both EDPAs and non-EDPA neighborhoods. However, changes occurred in the EDPA neighborhoods are larger than that happened in the non-EDPA neighborhoods. The employment rate of low income EDPA neighborhoods increased about 12.35\%, while it increased $10.34 \%$ in the non-EDPA neighborhoods. Employment rate in mediumand high-income neighborhoods decreased during the study period in both EDPAs and non-EDPAs. Generally, across all the three groups, the poverty level was higher in the EDPA neighborhoods than that in the non-EDPA neighborhoods and it changed more in the EDPA neighborhoods. Poverty level of the high-income neighborhood increased in both datasets but decreased in low- and medium-income EDPA neighborhoods. This indicates that formerly rich neighborhood experienced an influx of poor residents. Finally, we turn to the change of housing prices in both datasets. From Table 2 we noticed that no matter before or after the enterprise zone was designated, the deviations of house price for all the EDPA neighborhoods among all the three groups were smaller than the deviations for the non-EDPA neighborhoods. Comparing the change of housing prices for different income groups we found that low- and medium-income neighborhoods experienced price increase in both EDPAs and non-EDPAs, and low-income neighborhoods exhibited a larger increase. Housing price in high income EDPA neighborhoods decreased during our study 
period. All the facts showed above convince that changes of neighborhood characteristics are different among different income groups.

Table 2. 2000-2010 Changes of Outcome Variables in Neighborhoods with Different Initial Economic Status.

\begin{tabular}{|c|c|c|c|c|c|c|}
\hline \multirow{2}{*}{ Variable } & \multicolumn{3}{|c|}{ EDPAs } & \multicolumn{3}{|c|}{ Non-EDPAs } \\
\hline & 2000 & 2010 & Change (\%) & 2000 & 2010 & Change (\%) \\
\hline \multicolumn{7}{|l|}{ Population } \\
\hline Low & 185.4 & 215.9 & 14.13 & 199.8 & 198.1 & -0.851 \\
\hline Medium & 144.8 & 139.1 & -3.936 & 174.8 & 197.1 & 12.76 \\
\hline High & 143.5 & 166.9 & 16.31 & 145.6 & 163.6 & 12.36 \\
\hline \multicolumn{7}{|c|}{ Percent Black } \\
\hline Low & 0.958 & 0.865 & -9.708 & 0.916 & 0.812 & -11.35 \\
\hline Medium & 0.868 & 0.817 & -5.875 & 0.724 & 0.653 & -9.807 \\
\hline High & 0.819 & 0.787 & -3.907 & 0.169 & 0.185 & 9.467 \\
\hline \multicolumn{7}{|c|}{ Employment } \\
\hline \multicolumn{7}{|c|}{ Rate } \\
\hline Low & 0.413 & 0.464 & 12.35 & 0.435 & 0.480 & 10.34 \\
\hline Medium & 0.524 & 0.479 & -8.59 & 0.592 & 0.581 & -1.858 \\
\hline High & 0.726 & 0.598 & -17.63 & 0.700 & 0.672 & -4.000 \\
\hline \multicolumn{7}{|c|}{ Poverty Level } \\
\hline Low & 0.411 & 0.323 & -21.41 & 0.386 & 0.378 & -2.072 \\
\hline Medium & 0.230 & 0.205 & -10.87 & 0.201 & 0.217 & 7.960 \\
\hline High & 0.063 & 0.096 & 52.38 & 0.051 & 0.068 & 33.33 \\
\hline \multicolumn{7}{|c|}{$\begin{array}{l}\text { House Price } \\
\text { (USD 1000) }\end{array}$} \\
\hline Low & 73.28 & 84.86 & 13.65 & 85.43 & 106.3 & 19.63 \\
\hline Medium & 83.67 & 96.85 & 13.61 & 133.6 & 145.8 & 8.368 \\
\hline High & 126.9 & 115.4 & -9.965 & 282.8 & 309.4 & 8.597 \\
\hline
\end{tabular}

\subsection{Empirical Results}

When analyzing the treatment effects, the ideal situation is that participants are randomized into control and treatment groups, leading to the same chance of being assigned to the treatment group for each participant. In this way, differences in group means are only caused by treatment because groups can act as each other's counterfactual. However, in our analysis, there are some criteria for the neighborhoods being selected into the enterprise zone, and the counterfactual cannot be measured due to the nonrandomized selection. Therefore, the key challenge of evaluating the treatment effect of the EDPA designation in our study is how to get the counterfactual outcomes. To solve this problem, both propensity score weighting and Blinder-Oaxaca estimates are applied in this paper. Propensity score for each community is estimated using a logistic model. Table 3 provides the estimation results for the naïve DID, propensity score DID, and the Blinder-Oaxaca DID. The naïve DID estimation shows a significant $1.4 \%$ decrease in the fraction of the neighborhood that is employed, a $3.1 \%$ decrease in poverty rate, and a $4.5 \%$ increase in the housing price. The propensity score reweighting DID changed the magnitude (though not the sign) of the naïve DID estimates for poverty rate and housing price but found an insignificant effect on employment rate. The impact on poverty rate and housing price from propensity score DID increases to $5.9 \%$ and $7.2 \%$, respectively. For comparison we also report regression based on Blinder-Oaxaca estimates. Similar to the finding of propensity score DID, the Blinder-Oaxaca method yielded no significant effect on employment rate, a significant decrease in poverty rate, and a significant increase in housing price. In addition, the Blinder-Oaxaca estimator also finds a significant $9.4 \%$ increase in the median household income and a 5.9\% decrease in the fraction of residents that are black. Taken together the propensity score DID and the Blinder-Oaxaca DID, our results buttress the conclusion of much (but not all) of the literature that failed to find beneficial effects of enterprise zones in 
the United States for employment rate $[10,16,32,35]$. Though the lack of a significant effect on employment seems surprising in light of the EDPA program's incentives, economic theory provides some possible explanations for this finding. First, the incentive of the EDPA program is to hire disadvantaged workers. If there are opportunities to substitute low-skilled for higher-skilled labor, this incentive may induce a fair amount of labor-labor substitution, which would lead to weaker effect on overall employment [10]. The second possible explanation is that if enterprise zone program is targeting machinery and property, it could lead to substitution away from labor and towards other inputs, and therefore, the overall employment effect could be positive or negative. Consistent with Ham et al. (2011) [32], the estimates from all three approaches suggest that EDPA designation reduced poverty rate, and increased housing values.

Table 3. Impact of EDPAs on Neighborhoods—Difference-in-Difference (DID) Estimates.

\begin{tabular}{|c|c|c|c|c|}
\hline & & Naïve DID & Propensity Score DID & Blinder-Oaxaca DID \\
\hline \multirow[t]{3}{*}{ Log (population) } & Coeff. & 0.017 & -0.025 & 0.039 \\
\hline & CI & {$[-0.037,-0.071]$} & {$[-0.117,0.066]$} & {$[-0.009,0.087]$} \\
\hline & $p$-value & 0.531 & 0.587 & 0.114 \\
\hline \multirow[t]{3}{*}{$\%$ Black } & Coeff. & -0.019 & 0.124 & $-0.059^{* *}$ \\
\hline & CI & {$[-0.076,0.038]$} & {$[-0.128,0.376]$} & {$[-0.116,-0.002]$} \\
\hline & $p$-value & 0.516 & 0.333 & 0.044 \\
\hline \multirow[t]{3}{*}{ \% Employment } & Coeff. & -0.014 * & -0.017 & -0.009 \\
\hline & CI & {$[-0.029,0.000]$} & {$[-0.047,0.014]$} & {$[-0.022,0.004]$} \\
\hline & $p$-value & 0.054 & 0.284 & 0.175 \\
\hline \multirow[t]{3}{*}{ Log (income) } & Coeff. & -0.024 & 0.047 & $0.094^{* * *}$ \\
\hline & CI & {$[-0.082,0.035]$} & {$[-0.077,0.172]$} & {$[0.065,0.123]$} \\
\hline & $p$-value & 0.423 & 0.456 & 0.000 \\
\hline \multirow[t]{3}{*}{ Poverty Rate } & Coeff. & $-0.031^{* * *}$ & $-0.059^{* * *}$ & $-0.011 *$ \\
\hline & CI & {$[-0.039,-0.023]$} & {$[-0.090,0.029]$} & {$[-0.023,0.002]$} \\
\hline & $p$-value & 0.000 & 0.000 & 0.097 \\
\hline \multirow[t]{3}{*}{ Log (house price) } & Coeff. & $0.045^{* *}$ & $0.072^{* *}$ & 0.073 * \\
\hline & CI & {$[0.001,0.090]$} & {$[0.001,0.145]$} & {$[-0.009,0.154]$} \\
\hline & $p$-value & 0.048 & 0.046 & 0.082 \\
\hline
\end{tabular}

Note: Naïve DID refers to difference in difference estimates without covariate adjustments; propensity score matching DID was calculated using 2000 neighborhood characteristics; Blinder-Oaxaca DID computes counterfactual means of control neighborhoods in EDPAs via regression methods. ${ }^{* * *}$ indicates statistically significant at $1 \%, * *$ indicated statistically significant at $5 \%$, and ${ }^{*}$ indicates statistically significant at $10 \%$.

Table 4 provides estimations converting the estimated treatment effects from both propensity score DID and Blinder-Oaxaca DID in Table 3 into effects on totals. The calculations yield an estimated decrease of 3231 black individuals from the Blinder-Oaxaca estimates. The number of employed individuals increased by 1807 and 931 from the propensity score DID and Blinder-Oaxaca DID, respectively. Propensity score DID estimates indicated a decrease of 3231 individuals who were in poverty status, while Blinder-Oaxaca DID yielded a much smaller decrease of 602 individuals. By comparing changes in housing prices of EPDA neighborhoods to those of non-EPDA neighborhoods, we found that EDPA designation was estimated to increase the average house price by USD 6651 and USD 6742 from the propensity score DID and Blinder-Oaxaca DID, respectively. This is consistent with the hypothesis that the enterprise zone program could attract new business which would increase land value, and lead to the increase of local housing price.

Table 4. Impact Calculations.

\begin{tabular}{ccc}
\hline Panel A & Variable Values Inside EDPAs (2000) & \\
\hline & Total Population & 54,762 \\
& Average Household Income (U.S. Dollar) & 28,082 \\
& Average Housing Price (U.S. Dolalr) & 92,372 \\
\hline
\end{tabular}


Table 4. Cont.

\begin{tabular}{|c|c|c|c|}
\hline Panel B & Estimated Effects of the EDPAs & & \\
\hline & & Propensity Score DID & Blinder-Oaxaca DID \\
\hline & Decrease in black population between & 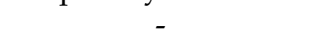 & 3231 \\
\hline & Decrease in poverty headcount & 3231 & 602 \\
\hline & Increase in average household income & - & 2640 \\
\hline & Increase in average housing price & 6651 & 6743 \\
\hline
\end{tabular}

Note: Values in Panel B are calculated using estimated coefficients from Table 3. Multiplying the variable values inside EDPAs in 2000. Statistically insignificant variables are omitted in Table 4.

\subsection{Heterogeneous Analysis}

As noted above, impact of enterprise zone programs would vary with initial neighborhoods economic status. Therefore, we next check the heterogeneous effects of the EDPA designation with respect to neighborhoods of different income level. Specifically, we divided the dataset into low-income neighborhoods, medium-income neighborhoods, and high-income neighborhoods, based on their 2000 median household income distribution $(25 \%, 75 \%$, and above). Estimation results from propensity score DID and Blinder-Oaxaca DID were described in Table 5. Both propensity score DID and Blinder-Oaxaca DID showed a relative larger and significant effect of EDPA on low income neighborhoods. Different from estimation results in Table 3, the heterogeneous analysis found a significant and positive effect of EDPA on employment of low-income neighborhoods, which indicated that the EDPA program incurred positive labor demand in these neighborhoods. With more people in the labor market, median household income in low-income neighborhoods increased and poverty rate decreased. Furthermore, Table 5 also showed that with positive labor demand shock caused by EDPA designation in low-income neighborhoods, more population migrated in these neighborhoods, which put upward pressure on housing prices.

Table 5. Robustness Estimates Based on Neighborhood Initial (2000) Economic Status.

\begin{tabular}{ccc}
\hline Variable & Propensity Score DID & Blinder-Oaxaca DID \\
\hline Log (population) & $0.042 *$ & $0.084 *$ \\
Low & $(-0.006,0.092)$ & $(-0.004,0.173)$ \\
Medium & -0.013 & -0.010 \\
High & $(-0.114,0.089)$ & $(-0.083,0.062)$ \\
\% Black & 0.071 & -0.043 \\
Low & $(-1.154,1.297)$ & $(-0.202,0.115)$ \\
Medium & 0.026 & -0.032 \\
High & $(-0.020,0.071)$ & $(-0.078,0.012)$ \\
\%Employment & 0.063 & $-0.054 *$ \\
Low & $(-0.035,0.161)$ & $(-0.110,0.002)$ \\
Medium & -0.002 & -0.016 \\
High & $(-0.021,0.015)$ & $(-0.047,0.015)$ \\
& $0.043 * *$ & $0.016 *$ \\
& $(0.011,0.075)$ & $(-0.002,0.034)$ \\
& 0.025 & 0.006 \\
& $(-0.011,0.061)$ & $(-0.017,0.027)$ \\
& 0.104 & 0.004 \\
& $(-1.199,1.409)$ & $(-0.016,0.023)$ \\
\hline
\end{tabular}


Table 5. Cont.

\begin{tabular}{ccc}
\hline Variable & Propensity Score DID & Blinder-Oaxaca DID \\
\hline Log (income) & $0.138^{* * *}$ & $0.135^{*}$ \\
Low & $0.043,0.232)$ & $(-0.014,0.283)$ \\
Medium & 0.002 & $0.127^{* * *}$ \\
High & $0.180,0.183)$ & $(0.056,0.196)$ \\
Poverty Rate & 0.232 & $(-0.167,0.121)$ \\
Low & $-1.110,1.576)$ & $-0.021^{*}$ \\
Medium & $(-0.124,-0.048)$ & $(-0.051,0.009)$ \\
High & $-0.046^{* *}$ & -0.002 \\
Log (house price) & $(-0.081,-0.011)$ & $(-0.019,0.016)$ \\
Low & -0.106 & 0.004 \\
Medium & $(-1.146,0.935)$ & $(-0.024,0.031)$ \\
High & $0.132 * * *$ & $0.095 *$ \\
& $(0.077,0.186)$ & $(-0.025,0.216)$ \\
& $0.052^{*}$ & -0.040 \\
& $(-0.046,0.160)$ & $(-0.052,0.132)$ \\
& -0.243 & 0.120 \\
& $(-3.182,2.696)$ & $(-0.356,0.596)$
\end{tabular}

Note: Only estimation results for propensity score DID and Blinder-Oaxaca DID are showed in Table 5. Confidence Intervals are provided in parentheses. ${ }^{* * *}$ indicates statistically significant at $1 \%,{ }^{* *}$ indicated statistically significant at $5 \%$, and * indicates statistically significant at $10 \%$.

In this study, we arbitrarily define the new neighborhoods as a set of 0.25 -milediameter circles and do not know whether the estimation results are sensitive to the neighborhood size. Since 0.25 -mile-diameter circles are already a very small unit to study the treatment effect of the EDPA designation, to verify the robustness of above results, we apply an alternative definition of the neighborhoods as larger size circles-0.5-milediameter circles. Then, the demographic data and housing price information are attached to the new neighborhoods using the same logic described above. With the 0.5 -mile-diameter neighborhoods, we replicated the estimation process, and the results are shown in Table 6 . Consistent with the 0.25 -mile-diameter neighborhood conclusions, 0.5 -mile-radius neighborhood estimates showed statistically significant effects of EDPA on poverty rate and housing price for all the three estimate approaches, but insignificant effect on population size and employment rate. Though the magnitudes of the average treatment effect from these sensitivity analyses are higher, the qualitative nature of the results does not change.

Table 6. Robustness Estimates Using 0.5-mile-diameter Neighborhoods.

\begin{tabular}{ccccc}
\hline & & Naïve DID & Propensity Score DID & Blinder-Oaxaca DID \\
\hline Log (population) & Coeff. & -0.020 & 0.145 & -0.049 \\
& CI & {$[-0.072,-0.031]$} & {$[-0.038,0.328]$} & {$[-0.192,0.094]$} \\
\% Black & $p$-value & 0.439 & 0.119 & 0.496 \\
& Coeff. & -0.047 & 0.035 & -0.023 \\
\% Employment & CI & {$[-0.114,0.019]$} & {$[-0.150,0.220]$} & {$[-0.086,0.039]$} \\
& $p$-value & 0.165 & 0.707 & 0.462 \\
Coeff. & CI & -0.002 & 0.009 & 0.003 \\
Log (income) & $p$-value & {$[-0.037,0.033]$} & {$[-0.054,0.072]$} & {$[-0.029,0.036]$} \\
& Coeff. & 0.905 & 0.777 & 0.845 \\
& CI & 0.004 & 0.089 & $0.059 *$ \\
& $p$-value & {$[-0.130,0.138]$} & {$[-0.069,0.248]$} & {$[-0.009,0.126]$} \\
\hline
\end{tabular}


Table 6. Cont.

\begin{tabular}{ccccc}
\hline & & Naïve DID & Propensity Score DID & Blinder-Oaxaca DID \\
\hline Poverty Rate & Coeff. & $-0.080 * * *$ & $-0.071^{*}$ & $-0.024^{*}$ \\
& CI & {$[-0.115,-0.045]$} & {$[-0.090,0.029]$} & {$[-0.053,0.004]$} \\
Log (house price) & -value & 0.000 & 0.060 & 0.093 \\
& Coeff. & $0.065 *$ & $0.084^{*}$ & $0.092^{*}$ \\
& CI & {$[0.002,0.128]$} & {$[-0.146,0.003]$} & {$[-0.002,0.186]$} \\
& $p$-value & 0.043 & 0.068 & 0.054 \\
\hline
\end{tabular}

Note: Naïve DID refers to difference in difference estimates without covariate adjustments; propensity score matching DID was calculated using 2000 neighborhood characteristics; Blinder-Oaxaca DID computes counterfactual means of control neighborhoods in EDPAs via regression methods. ${ }^{* * *}$ indicates statistically significant at $1 \%$, and * indicates statistically significant at $10 \%$.

\section{Discussion}

In this paper, we exploited the unique nature of Atlanta's EDPA program to estimate its effects at neighborhood level. The statistical analysis showed that the EDPA neighborhoods had small population size, high levels of poverty, low-income growth, and high unemployment. This confirmed the primary goals of Atlanta's EDPA to "create jobs for disadvantaged communities" and "revitalize the economically distressed communities". All three estimation strategies found a poverty reduction effect (3.1\% from naïve DID, 5.9\% from propensity score DID, and $1.1 \%$ from the Blinder-Oaxaca DID) of the EDPA program. This finding is consistent with Busso and Kline (2008) [12] and Hanson (2009) [36], which showed negative effects of the federal enterprise zone programs on poverty rates. However, we did not get a significant effect of EDPA designation on employment rate. The possible explanation for this result may be the lack of wage tax credit, so that firms do not have to expand employment to realize benefit.

Regarding property value, our results also showed neighborhoods within EDPAs paid more (7.2\% from propensity score DID and 7.3\% from the Blinder-Oaxaca DID) for their property compared to those outside the zones. This result should be encouraging to the city as the increased housing prices translate into additional property tax revenue, which indicates that Atlanta may lower tax rates to increase revenue through property taxes. This policy implication is similar to Skidmore et al. (2012) [37]. The increased housing prices could be explained by the following reasons. First, the EDPA designation increased competition for the houses in affected neighborhoods, which has driven up the price. Related to increased demand, there may also be a decrease in demand for nonEDPA neighborhoods [1]. That is, it is possible these zones create a greater number of vacancies and less attractive neighborhoods in areas outside EDPAs as residents leave for or are only attracted to more desirable EDPA neighborhoods that have lower tax rates and positive externalities. Second, implementing the EDPA may correspond with other city efforts to bolster these neighborhoods. Perhaps the city provides more, or better, public services to EDPA neighborhoods to keep the area thriving. The heterogeneous analysis indicated that the initial characteristics of neighborhood did matter for the effectiveness of the EDPA program. Therefore, when providing tax abatements, initial economic status of the neighborhoods should be paid a little bit more attention.

Finally, although we found poverty reduction effect from the EDPA program in Atlanta, it would be misguided to assume similar effects occur in other areas implementing place-based policies. Regarding the increased housing prices due to EDPA designation from our analysis, it is unknown what effect this program has had on vacancy and mobility rates, and thus the benefits may continue to be under- or overestimated.

\section{Conclusions}

Recently, a growing body of theoretical and empirical studies has examined the question whether place-based policies are effective in promoting regional growth. This study provides an empirical analysis of the impact of Atlanta's EDPA program on neighborhood population size and composition, employment rate, median household income, poverty 
rate, and housing price. Apart from existing literature applying census block groups or census tracts as the study unit, we created a set of 0.25-mile-diameter circles (alternatively 0.5-mile-diameter circles) evenly distributed across Atlanta, and defined these circles as our neighborhoods. This circle method avoids us adjusting the change of census block group or census tract boundaries during the study period. The analysis focused on the change of neighborhood characteristics of Atlanta, GA, between 2000 (pre-program) and 2010 (post-program). To do the pre-program and post-program comparison of DEPA neighborhoods and non-EDPA neighborhoods, the naïve DID, propensity score weighted DID and Blinder-Oaxaca DID approaches were applied in this paper.

Our comparison of EDPA neighborhoods to non-EDPA neighborhoods showed a poverty reduction effect of Atlanta's EDPA designation but failed to find beneficial effects of on population size and employment rate. Though this conclusion is contradicted by the research of Ham et al. (2011) [32] showing that state and federal enterprise zones generated large labor market benefits, it is consistent with much of the broader literature suggesting enterprise zones had no effect on employment [10,16,32]. Our conclusion of the growth in housing values is consistent with previous studies finding positive effect of enterprise zones on housing price. Engberg and Greenbaum (1999) [23] suggested enterprise zone program induced increase in house demand and therefore prompted a price rise, especially in places that with low vacancy rates during the pre-program period. Our heterogeneous analysis results with respect to neighborhoods' initial economic status appeared to corroborate the findings of Guerrieri et al. (2012) [34]. Estimation results for propensity score DID and Blinder-Oaxaca DID showed a relative larger and significant effect of EDPA designation on low income neighborhoods, and the heterogeneous analysis found a significant and positive effect on employment of low income neighborhoods, which indicated that the EDPA program is more beneficial to disadvantaged neighborhoods. With increasing labor demand induced by EDPA designation in low-income neighborhoods, our research also showed that more population migrate in these neighborhoods, putting upward pressure on housing prices. Our robustness estimates of 0.5 -mile circle neighborhoods are consistent with the 0.25 -mile circle neighborhoods estimates.

Author Contributions: Conceptualization, Y.L., Z.Z. and J.Z.; methodology, Y.L. and Z.Z.; validation, Y.L., Z.Z. and D.H.; formal analysis, Y.L. and J.Z.; writing—original draft preparation, Y.L. and Z.Z.; writing-review and editing, D.H. and J.Z.; supervision, D.H. All authors have read and agreed to the published version of the manuscript.

Funding: This research was funded by the National Social Science Foundation of China (19BGL199, 19BGL160), the Humanities and Social Research Project of Ministry of Education of China (17YJCZH091), the Research Team for High Quality of Economic Development of Shandong Province (2020RWE009), and the Philosophy and Social Science Project of Shandong Province (19DGLJ09).

Institutional Review Board Statement: Not applicable.

Informed Consent Statement: Not applicable.

Data Availability Statement: Not applicable.

Conflicts of Interest: The authors declare no conflict of interest.

\section{References}

1. Hodge, T.R.; Komarek, T.M. Capitalizing on Neighborhood Enterprise Zones: Are Detroit residents paying for the NEZ homestead exemption? Reg. Sci. Urban Econ. 2016, 61, 18-25. [CrossRef]

2. Kline, P.M.; Moretti, E. Local economic develpment, agglomertation economices, and the big push. Q. J. Econ. 2014, 129, $275-331$. [CrossRef]

3. Givord, P.; Quantin, S.; Trevien, C. A long-term evaluation of the first generation of French urban enterprise zones. J. Urban Econ. 2018, 105, 149-161. [CrossRef]

4. Neumark, D.; Simpson, H. Place-Based Policies, 1st ed.; Elsevier: Amsterdam, The Netherlands, 2015; Volume 5, ISBN 9780444595317.

5. Jia, J.; Ma, G.; Qin, C.; Wang, L. Place-based policies, state-led industrialisation, and regional development: Evidence from China's Great Western Development Programme. Eur. Econ. Rev. 2020, 123, 103398. [CrossRef] 
6. Kline, P.; Moretti, E. People, places and public policy: Some simple welfare economics of local economic development programs. Annu. Rev. Econ. 2014, 6, 629-662. [CrossRef]

7. Shenoy, A. Regional development through place-based policies: Evidence from a spatial discontinuity. J. Dev. Econ. 2018, 130, 173-189. [CrossRef]

8. Gobillon, L.; Magnac, T.; Selod, H. Do unemployed workers benefit from enterprise zones? The French experience. J. Public Econ. 2012, 96, 881-892. [CrossRef]

9. O'Keefe, S. Job creation in California's enterprise zones: A comparison using a propensity score matching model. J. Urban Econ. 2004, 55, 131-150. [CrossRef]

10. Neumark, D.; Kolko, J. Do enterprise zones create jobs? Evidence from California's enterprise zone program. J. Urban Econ. 2010, 68, 1-19. [CrossRef]

11. Bondonio, D.; Engberg, J. Enterprise zones and local employment: Evidence from the states' programs. Reg. Sci. Urban Econ. 2000, 30, 519-549. [CrossRef]

12. Busso, M.; Kline, P.; Haven, N. Do Local Economic Development Programs Work? Evidence from the Federal Empowerment Zone Program. Cowles Foundation Discussion Paper; Cowles Foundation for Research in Economics, Yale University: New Haven, CT, USA, 2008.

13. Lambert, T.E.; Coomes, P.A. An evaluation of the effectiveness of Louisville's Enterprise Zone. Econ. Dev. Q. 2001, 15, 168-180. [CrossRef]

14. Zhang, S. Impacts of Enterprise Zone Policy on industry growth: New evidence from the Louisville Program. Econ. Dev. Q. 2015, 29, 347-362. [CrossRef]

15. Falck, O.; Koenen, J.; Lohse, T. Evaluating a place-based innovation policy: Evidence from the innovative Regional Growth Cores Program in East Germany. Reg. Sci. Urban Econ. 2019, 79, 103480. [CrossRef]

16. Faggio, G. Relocation of public sector workers: Evaluating a place-based policy. J. Urban Econ. 2019, 111, 53-75. [CrossRef]

17. Neumark, D.; Young, T. Enterprise zones, poverty, and labor market outcomes: Resolving conflicting evidence. Reg. Sci. Urban Econ. 2019, 78, 103462. [CrossRef]

18. Glaeser, E.L.; Gottlieb, J.D. The economics of place-making policies. Brook. Pap. Econ. Act. 2008, 39, 155-253. [CrossRef]

19. Einiö, E.; Overman, H.G. The (Displacement) Effects of Spatially Targeted Enterprise Initiatives: Evidence from UK LEGI; SERC Discussion Papers 0191; Spatial Economics Research Centre, LSE: London, UK, 2016; Volume 191, p. 43.

20. Billings, S. Do enterprise zones work?: An analysis at the borders. Public Financ. Rev. 2009, 37, 68-93. [CrossRef]

21. Brachert, M.; Dettmann, E.; Titze, M. The regional effects of a place-based policy-Causal evidence from Germany. Reg. Sci. Urban Econ. 2019, 79. [CrossRef]

22. Landers, J. Why don't enterprise zones work? Estimates of the extent that EZ benefits are capitalized into property values. J. Reg. Anal. Policy 2006, 36, 15-30.

23. Engberg, J.; Greenbaum, R. State enterprise zones and local housing markets. J. Hous. Res. 1999, 10, 163-187. [CrossRef]

24. Banzhaf, H.S.; Walsh, R.P. Do people vote with their feet? An empirical test of Tiebout's mechanism. Am. Econ. Rev. 2008, 98, 843-863. [CrossRef]

25. Splawa-Neyman, J. On the application of probability theory to agricultural experiments. Essay on Principles. Section 9. Stat. Sci. 1990, 5, 465-472. [CrossRef]

26. Rubin, D. Estimating causal effects of treatments in randomized and nonrandomized studies. J. Educ. Psychol. 1974, 66, 688-701. [CrossRef]

27. Blinder, A. Wage discrimination: Reduced form and structural estimates. J. Hum. Resour. 1973, 8, 436-445. [CrossRef]

28. Oaxaca, R. Male-female wage differentials in urban labor markets. Int. Econ. Rev. 1973, 14, 693-709. [CrossRef]

29. Horvitz, D.G.; Thompson, D.J. A generalization of sampling without replacement from a finite universe. J. Am. Stat. Assoc. 1952, 47, 663-685. [CrossRef]

30. Heckman, J.; Ichimura, H.; Smith, J.; Todd, P. Characterizing selection bias using experimental data. Econometrica 1998, 66, 1017-1098. [CrossRef]

31. Rosenbaum, P.R.; Rubin, D.B. The central role of the propensity score in observational studies for causal effects. Biometrika 1983 70, 41-55. [CrossRef]

32. Ham, J.C.; Swenson, C.; İmrohorğlu, A.; Song, H. Government programs can improve local labor markets: Evidence from State Enterprise Zones, Federal Empowerment Zones and Federal Enterprise Community. J. Public Econ. 2011, 95, 779-797. [CrossRef]

33. Birch, C.P.D.; Oom, S.P.; Beecham, J.A. Rectangular and hexagonal grids used for observation, experiment and simulation in ecology. Ecol. Model. 2007, 206, 347-359. [CrossRef]

34. Guerrieri, V.; Hartley, D.; Hurst, E. Within-City Variation in Urban Decline: The Case of Detroit. Am. Econ. Rev. Pap. Proceeding 2012, 102, 120-126. [CrossRef]

35. Elvery, J.A. The Impact of Enterprise Zones on Resident Employment: An Evaluation of the Enterprise Zone Programs of California and Florida. Econ. Dev. Q. 2008, 23, 44-59. [CrossRef]

36. Hanson, A. Local employment, poverty, and property value effects of geographically-targeted tax incentives: An instrumental variables approach. Reg. Sci. Urban Econ. 2009, 39, 721-731. [CrossRef]

37. Skidmore, M.; Reese, L.; Kang, S.H. Regional analysis of property taxation, education finance reform, and property value growth Reg. Sci. Urban Econ. 2012, 42, 351-363. [CrossRef] 